\title{
Self-perceived stress is associated with adiposity and atherosclerosis. The GEA Study
}

\author{
Janinne Ortega-Montiel, Carlos Posadas-Romero, Wendy Ocampo-Arcos, Aida Medina-Urrutia, \\ Guillermo Cardoso-Saldaña, Esteban Jorge-Galarza and Rosalinda Posadas-Sánchez
}

\begin{abstract}
Background: A growing body of evidence suggests that psychological stress is an independent cardiovascular risk factor. Obesity prevalence shows accelerating trends worldwide, and is known to be associated with a range of comorbidities and survival. The aim of this study was to assess the relationship between self-perceived psychological stress with parameters of adiposity, metabolic syndrome, and subclinical atherosclerosis in Mexican participants.

Methods: Metabolic Syndrome was defined using the Adult Treatment Panel III criteria, obesity was defined as $\mathrm{BMI}>30$, subclinical atherosclerosis disease was determined by computed tomography, and carotid intima media thickness was determined by ultrasonography. Self-perceived psychological stress was assessed using a single-item questionnaire.

Results: A total of 1243 control subjects were included in the sample, mean age $54.2 \pm 9$ years old; the prevalence of chronic self-perceived psychological stress (>5 years) was $10.13 \%$, female gender ( $62.7 \%$ ), obesity prevalence $(48.4 \%)$, and self-reporting sedentary lifestyle (56.3\%). The chronic stressed cohort presented higher subcutaneous abdominal fat content (285 vs $319 \mathrm{~cm}^{2}$ ), and carotid intima media thickness ( 0.63 vs $0.66 \mathrm{~mm} ; \mathrm{p}<0.01$ for both). However, after adjustment for lifestyle/social covariates (Model 1) and biological mediators (Model 2), chronic self-perceived stress was independently associated with obesity in men (OR 2.85, $95 \% \mathrm{Cl} 1.51-5.40)$ and carotid atherosclerosis in women (OR 2.262, $95 \% \mathrm{Cl} 1.47-4.67 ; \mathrm{p}<0.01$ for both).
\end{abstract}

Conclusion: Our study suggests that self-reported chronic stress is an independent risk factor for obesity in men. In addition, carotid atherosclerosis was also found to be an independent risk factor in women in a Mexican population sample.

\section{Background}

The increasing obesity prevalence is a major public health problem and it is associated with chronic health conditions such as coronary heart disease, type 2 diabetes mellitus (T2DM), and metabolic syndrome (MS) [1]. The etiology of obesity is multifaceted, involving interactions between individuals and their social, cultural, and physical environments. Psychosocial stress has also been considered as a potential factor for obesity, leading

\footnotetext{
*Correspondence: rossy_posadas_s@yahoo.it

Department of Endocrinology, National Institute of Cardiology "Ignacio Chavez", Juan Badiano No. 1 Col. Seccion XVI, Tlalpan, 14080 Mexico City, Mexico
}

\section{() Biomed Central}

(C) 2015 Ortega-Montiel et al. Open Access This article is distributed under the terms of the Creative Commons Attribution 4.0 International License (http://creativecommons.org/licenses/by/4.0/), which permits unrestricted use, distribution, and reproduction in any medium, provided you give appropriate credit to the original author(s) and the source, provide a link to the Creative Commons license, and indicate if changes were made. The Creative Commons Public Domain Dedication waiver (http://creativecommons.org/publicdomain/zero/1.0/) applies to the data made available in this article, unless otherwise stated. to a growing pool of clinical and epidemiological data [2]. In a number of publications, several psychosocial factors such as depression, anxiety, chronic stress, hopelessness, and certain behavioral patterns, have been linked to the development of metabolic syndrome and atherosclerosis [3]. A recent meta-analysis of longitudinal studies confirmed that psychological stress is positively related to the development of adiposity in prospective studies, although the effects were modest [4]. Stress is an ubiquitous term with no universally accepted definition. For scientific purposes, Gold and McEwen in 1998 defined "stress" as a state of threatened homeostasis; and "chronic stress" as the long-term repeated disruption of the homeostasis 
system. The human stressors are generally complex life events such as adversity in a relationship, overall health, work, and personal finances [5].

A growing body of literature has now provided convincing evidence that the underlying mechanisms of pathophysiological stress include inflammatory and immune processes, alterations in activating hypothalamic pituitary adrenal axis, and alterations in autonomic nervous system activity, among others. The impact of environmental factors, such as increased life stress and endogenous depression has also been linked to pathophysiological stress [5]. Cortisol hyper-secretion has been associated with a variety of diseases such as hypertension, osteoporosis, depression, and metabolic syndrome components, which include visceral obesity, dyslipidemia, and insulin resistance [6]. Such diseases have also been considered as risk factors for cardiovascular morbidity and mortality derived from atherosclerosis disease in adult populations [7]. Obesity, as the principal etiology of these metabolic disorders, has also been associated with frequent and/or persistent stimulation of the HPA axis and the accumulation of visceral fat, as a result of excessive corticosteroid [8]; thus, highlighting the importance of visceral and subcutaneous abdominal fat as a pathological mechanism of atherosclerosis disease. Psychological stress has also been considered an important risk factor for cardiovascular disease, in addition to stroke or myocardial infarction [9]. Epidemiological studies, like the INTERHEART Study [10], have used psychological stress questions, to assess subjective and perceived stressors to determine the prevalence and the impact of psychological stress. It has previously been found that chronic stress, but not periodic stress as assessed by this measure, can predict myocardial infarction and overall stroke in prospective studies [11]. Since then, several population-based studies have shown an association between psychological stress and increased risk of obesity and cardiovascular disease; however, risk estimates vary among studies, which could be due to differences in study design, ethnicity, sample size, age and gender, and different selfperceived stress measurements. Therefore, the purpose of the present study was to investigate the association of the frequency of self-perceived psychological stress with obesity, metabolic syndrome, and subclinical atherosclerosis disease in a Mexican population sample.

\section{Methods}

\section{Subjects}

The Genetics of Atherosclerosis Disease [Genetica de la Enfermedad Aterosclerosa] (GEA) Study was designed to investigate genetic risk factors associated with premature coronary heart disease, subclinical atherosclerosis, and other coronary risk factors in the Mexican mestizo population. A total of 2740 subjects, 1500 control subjects and 1240 coronary patients, aged $30-75$ years took part in the study. The participants were not related and self-reported Mexican mestizo ancestry. All control subjects were apparently healthy asymptomatic individuals, without family history of premature coronary artery disease (CAD), recruited from blood bank donors and through brochures posted in social services centers. The coronary patient group was recruited from the National Institute of Cardiology "Ignacio Chavez" (INCICH) using their medical history. Coronary patients and control subjects with a history of renal, liver, thyroid, or malignant disease, as well as those undergoing treatment with corticosteroids, were excluded [12]. For the purpose of the present study, only the control group was included. The GEA study was approved by the Institutional Review Board of the National Institute of Cardiology "Ignacio Chavez" and the Ethics Committee of the National Institute of Genomic Medicine (INMEGEN). The study was conducted according to the Declaration of Helsinki. Written informed consent was obtained from all participants. We excluded 257 subjects with unavailable responses of the self-perceived psychological stress item. The final study population for this analysis comprised 1243 subjects. In order to determine population differences between the included participants and those excluded with no self-perceived psychological stress responses, we compared the sociodemographic and biological parameters. Statistical analyses indicated that patients in the excluded group were younger ( 54.2 vs 49.2 years old; $\mathrm{p}<0.01$ ), and no other differences were observed.

\section{Data collection}

All participants answered a standardized and validated questionnaire to obtain information on family and medical history, cardiovascular risk factors, alcohol intake and smoking history, dietary habits [13], and physical activity [14]. The questionnaire applied to the entire population included a single-item question that has been used in epidemiological studies since 1970 [9-11], in order to evaluate the prevalence of self-perceived psychological stress in the studied population. In this question, stress is described as feeling tense, irritable, anxious, or as having sleep difficulties as a result of circumstances at home or at work. Participants were asked to report how often they had experienced stress, using the following incremental or graded response options: 1) Never, 2) At some point, 3) at some point during the last 5 years, 4 ) at several periods during the last 5 years, 5) Chronic stress during the last year, or 6) Chronic stress during the last 5 years.

\section{Anthropometric measures and risk factors definition}

Trained personnel measured anthropometric parameters, these included waist circumference (WC) and body mass index (BMI) - calculated as weight in kilograms divided by the square of height in meters. Blood pressure 
was measured three times by sphygnomannometry and the last two measurements were averaged. Obesity was defined as $B M I \geq 30 \mathrm{~kg} / \mathrm{m}^{2}$. Hypoalphalipoproteinemia, hypertriglyceridemia and metabolic syndrome were defined using the Adult Treatment Panel III criteria [15], except for the cut point of waist circumference [16]. Hypercholesterolemia was defined as total cholesterol levels $\geq 200 \mathrm{mg} / \mathrm{dl}$. Hypertension was defined as systolic blood pressure $\geq 140 \mathrm{mmHg}$ and/or diastolic blood pressure $\geq 90 \mathrm{mmHg}$, or the use of oral antihypertensive therapy; T2DM was diagnosed according to World Health Organization criteria [17]. Smoking history was coded as current versus never or former (smoking cessation at least one month before inclusion in the study); alcohol intake was defined as consumption in the last 6 months (at least one time per month) of any type of cocktail; sedentary lifestyle was defined by an item that considered whether the majority of the participant's free time was spent reading, watching TV or resting, and not practicing any physical activity. Chronic, but not periodic stress, as assessed by the single-item questionnaire, has been previously found to be associated with cardiovascular risk [9], crude OR was calculated between obesity and the different responses of the selfperceived psychological stress item, and only the score 6 (chronic stress during the last 5 years) showed significant association to an increased risk of obesity.

\section{Biochemical measures}

Venous blood samples were obtained after a 12-hour fasting period and all measurements were performed at the Laboratory of Endocrinology located at the INCICH, using standardized procedures. Accuracy and precision of lipid measurements in our laboratory are under periodic monitoring by the Centers for Disease Control and Prevention (Atlanta, GA, USA). Plasma glucose, total cholesterol (TC), triglyceride (TG), and High-density lipoprotein cholesterol (HDL-C) levels were measured with commercially available standardized methods. Low-density lipoprotein cholesterol (LDL-C) concentrations were estimated using Friedewald's formula modified by De Long [18]. Homeostasis model assessment of insulin resistance (HOMA-IR) was calculated from fasting glucose and insulin measures [19].

\section{Tomography measures}

Computed tomography of the chest and abdomen were performed using a 64-channel multi-detector helical computed tomography system (Somatom Sensation, Siemens) and interpreted by experienced radiologists. Scans were read to assess and quantify the following: 1) coronary artery calcification (CAC) score using the Agatston method [20]; 2) total abdominal, subcutaneous, and visceral adipose tissue areas, as described by Kvist [21], in order to calculate visceral adipose tissue/subcutaneous adipose tissue ratio (VAT/SAT); and 3) hepatic to splenic attenuation ratio (LSAR) as described by Longo et al. [22].

\section{Carotid artery intima media thickness}

Carotid intima-media-thickness measurements were performed using a MicroMaxx ultrasound device (SonoSite, Inc., Bothell, WA, USA), equipped with a 7.0-14 MHz linear-array wideband transducer to obtain B-mode ultrasound images [23]. The common carotid artery, bulb and internal carotid artery were bilaterally scanned over a length of $10 \mathrm{~mm}$. The mean combined outcome of the six segments was reported. The intra- and inter-observer correlation for IMT and total area using this method were 0.98 and 0.96 respectively. The cut points of IMT in Hispanic population were taken from the Ase Consensus Statement of the Society for Vascular Medicine [23].

\section{Statistical analyses}

All analyses were performed using SPSS version 18.0 (SPSS, Chicago, II) statistical package. The self-perceived psychological stress variable was dichotomized by the subjects who reported chronic self-perceived psychological stress for $>5$ years in one category (Score 6), and scores 1 thru 5 composed the reference category. Data are expressed as median (interquartile range) or as frequencies for categorical variables. Comparisons were made using the t-test or the Mann-Whitney U test, and by Chi square analysis for categorical variables. Logistic regression was used to determine the independent association between chronic self-perceived psychological stress with metabolic syndrome, parameters of obesity, and subclinical atherosclerosis. Stepwise adjustments were made, starting with unmodifiable and lifestyle/social risk factors (Model 1): Age, current smoking status, alcohol intake, physical activity, and marital status. Thereafter, further adjustments for known mediating biological risk factors (Systolic BP, LDL-C, HDL-C, TG and glucose) (Model 2) were added. The values of $\mathrm{p}<0.05$ were considered statistically significant.

\section{Results}

A total of 1243 subjects, mean age $54.2 \pm 9.0$ years ( $67.2 \%$ female), were included in the analysis. We found that $10.13 \%$ of the subjects reported chronic stress related to circumstances at work or home during the past 5 years. Demographic characteristics and prevalence of cardiovascular risk factors according to the dichotomized categories of self-perceived psychological stress are shown in Table 1. Compared with the no chronic stress participants, the chronic stress subjects had a significantly higher prevalence of sedentary lifestyle, obesity, and increased IMT (56.3\%, $48.4 \%$ and $37.5 \%$ respectively). We also found a higher proportion of females in the chronic stress participants, compared to the 
Table 1 Baseline, anthropometric, biochemical and tomographic characteristics by categories of self-perceived psychological stress

\begin{tabular}{llll}
\hline Risk factors & Never/periods & $\begin{array}{l}\text { Chronic } \geq 5 y \\
n=1117\end{array}$ & P value \\
\hline Female n (\%) & $555(49.7)$ & $79(62.7)$ & \\
Current smokers n (\%) & $242(21.7)$ & $30(23.8)$ & 0.006 \\
Alcohol intake n (\%) & $119(10.7)$ & $12(9.5)$ & 0.581 \\
Sedentary lifestyle n (\%) & $498(44.6)$ & $71(56.3)$ & 0.805 \\
Hypertension n (\%) & $222(19.9)$ & $21(16.7)$ & 0.012 \\
Diabetes n (\%) & $172(15.4)$ & $16(12.7)$ & 0.423 \\
Dyslipidemia n (\%) & $183(16.4)$ & $24(19)$ & 0.422 \\
Metabolic syndrome n (\%) & $480(43)$ & $61(48.4)$ & 0.243 \\
Physical Inactivity n (\%) & $500(44.8)$ & $72(57.1)$ & 0.008 \\
Overweight n (\%) & $538(48.2)$ & $48(38.1)$ & 0.024 \\
Obesity n (\%) & $354(31.7)$ & $61(48.4)$ & 0.001 \\
Increased IMT n (\%) & $170(22)$ & $42(37.5)$ & $<0.001$
\end{tabular}

Anthropometric and Biochemical

\begin{tabular}{|c|c|c|c|}
\hline Age (years) & $54.2(53.7-54.8)$ & $53.4(52.0-54.9)$ & 0.417 \\
\hline Waist circumference (cm) & $95.5(87.7-102.5)$ & $96(89-106.9)$ & 0.214 \\
\hline $\mathrm{BMI}\left(\mathrm{kg} / \mathrm{m}^{2}\right)$ & $28(25.5-31.1)$ & $29.8(26.4-32.3)$ & 0.002 \\
\hline Systolic BP (mmHg) & $114(105-126)$ & $114(102-123)$ & 0.353 \\
\hline $\mathrm{TC}(\mathrm{mg} / \mathrm{dl})$ & $191.8(166-215)$ & $190(166.6-208.9)$ & 0.725 \\
\hline LDL-C (mg/dl) & $117(95.6-137.7)$ & $114(97-137)$ & 0.571 \\
\hline $\mathrm{HDL}-\mathrm{C}(\mathrm{mg} / \mathrm{dl})$ & $44(36.3-54.3)$ & $42.6(35.1-51)$ & 0.309 \\
\hline $\mathrm{TG}(\mathrm{mg} / \mathrm{dl})$ & $149(113-205)$ & $152(109-202)$ & 0.712 \\
\hline Glucose (mg/dl) & $91(85-100)$ & $91(83-102)$ & 0.684 \\
\hline HOMA-IR & $4.1(2.7-6)$ & $4.2(2.9-6)$ & 0.628 \\
\hline Insulin $(\mu \mathrm{U} / \mathrm{ml})$ & $17.4(12.7-24.2)$ & $18.2(14-23.1)$ & 0.510 \\
\hline C-reactive protein (mg/dl) & $1.62(0.82-3.36)$ & $1.42(0.87-3.37)$ & 0.877 \\
\hline ALT (IU/L) & $24(18-34)$ & $26(19-35)$ & 0.106 \\
\hline AST (IU/L) & $25(21-31)$ & $25(22-32)$ & 0.120 \\
\hline \multicolumn{4}{|c|}{ Tomographic and ultrasonography } \\
\hline $\operatorname{TAF}\left(\mathrm{cm}^{2}\right)$ & $443(359-547)$ & $480(377-583)$ & 0.035 \\
\hline $\operatorname{SAF}\left(\mathrm{cm}^{2}\right)$ & $285(218-366)$ & $319(242-409)$ & 0.008 \\
\hline $\operatorname{VAF}\left(\mathrm{cm}^{2}\right)$ & $153(115-200)$ & 156 (106-192) & 0.706 \\
\hline IMTave (mm) & $0.63(0.55-0.73)$ & $0.66(0.60-0.83)$ & $<0.001$ \\
\hline
\end{tabular}

Differences between groups with the Chi square test for proportions and U-Mann Whitney test for median (range). IMT = Intima media thickness. BMI = Body mass index, $\mathrm{BP}=\mathrm{Blood}$ pressure, $\mathrm{TC}=$ Total cholesterol, $\mathrm{LDL}=\mathrm{L}$.w density lipoprotein, $\mathrm{HDL}=$ High-density lipoprotein, $\mathrm{TG}=$ Triglycerides, $\mathrm{HOMA}-\mathrm{IR}=$ Homeostasis model assessment of insulin resistance, $A L T=$ Alanine transaminase, $A S T=$ Aspartate transaminase, $\mathrm{TAF}=$ Total abdominal fat, $\mathrm{SAF}=$ Subcutaneous abdominal fat, $\mathrm{VAF}=$ Visceral abdominal fat and IMTave $=$ Intima media thickness average

non-chronic stress group. The anthropometric, biochemical, and tomographic characteristics of the groups are given in Table 1.

The subjects with chronic self-perceived stress showed higher BMI, total abdominal and subcutaneous abdominal fat, and carotid IMT average when compared with their counterparts. No significant differences were observed in the biochemical characteristics, including lipid profile, insulin, HOMA-IR, hepatic enzymes, and Creactive protein. When all these characteristics were analyzed by gender (data not shown), chronic-stressed men were younger and had elevated systolic BP compared to those without stress; also chronic-stressed women presented lower HDL-C, and higher AST levels compared to those without stress. Moreover, chronic-stressed women showed the greatest carotid intima media thickness.

Logistic regression models were used to assess whether self-perceived chronic stress was associated with metabolic syndrome or its individual components, obesity, and carotid atherosclerosis (Table 2). Compared with nonchronic stress females, the chronic stress female cohort were more likely to have hypoalphalipoproteinemia, independent of age, current smoking status, alcohol intake, physical activity, and marital status (Model 1). However, after full adjustment the association was no longer independent. The chronic stress women showed an independent association with increased IMT (Model 2), this association not only remained significant after full adjustment for confounding factors, the association even increased. In the chronic stress men cohort, there were no significant associations for the metabolic syndrome and its individual components. However, we found a significant association with obesity in both models.

\section{Discussion}

Our findings showed an independent association between chronic self-perceived stress (measured by a single-item questionnaire) and obesity, in males; and carotid atherosclerosis in female patients, which was previously reported to be linked to ischemic stroke [9].

We also showed an association between chronic stress and carotid atherosclerosis in women. Chronic hypersecretion of stress mediators such as cortisol, in individuals with genetic predisposition exposed to a permissive environment [24], is an established mechanism to the development of hypertension, dyslipidemia, type 2 diabetes mellitus, and atherosclerosis disease [25, 26]. Our results are supported by a large population sample study that investigated the association between psychological stress and carotid atherosclerosis. Our findings indicated that high psychological stress and carotid plaques were independently and linearly related, with plaque prevalence odds of 1.55 (95\% CI 1.10 to $2.18, \mathrm{p}=0.011$ ) among women and 1.38 (95\% CI 1.12 to $1.75, \mathrm{p}=0.005$ ) among men [27].

In this study, an independent association to chronic self-perceived stress was only found for sedentary lifestyle and BMI, as cardiovascular risk factors in the Mexican population. However, another study that investigated the relationship between stress at work and cardiovascular 
Table 2 Odds ratio (OR) of chronic self-perceived psychological stress for metabolic risk factors and subclinical atherosclerosis by gender

\begin{tabular}{|c|c|c|c|c|}
\hline \multirow[t]{3}{*}{ Metabolic risk factors } & \multicolumn{2}{|l|}{ Female $n=79$} & \multicolumn{2}{|l|}{ Male $n=47$} \\
\hline & OR (95\% Cl) P value & OR (95\% Cl) P value & OR (95\% Cl) P value & OR (95\% Cl) P value \\
\hline & Model 1 & Model 2 & Model 1 & Model 2 \\
\hline Systemic hypertension & $0.66(0.32-1.37) 0.27$ & $0.59(0.28-1.26) 0.17$ & $1.12(0.52-2.40) 0.75$ & $0.95(0.43-2.06) 0.90$ \\
\hline Hyper trigliceridemia & $1.31(0.81-2.12) 0.27$ & $0.61(0.29-1.29) 0.20$ & $0.89(0.48-1.66) 0.72$ & $0.94(0.43-2.05) 0.88$ \\
\hline Hypoalpha lipoproteinemia & $2.13(1.26-3.50) 0.004$ & $1.33(0.80-2.20) 0.26$ & $0.92(0.50-1.71) 0.81$ & $0.71(0.37-1.36) 0.30$ \\
\hline Diabetes Mellitus & $0.81(0.38-1.71) 0.58$ & $0.74(0.34-1.60) 0.45$ & $0.98(0.41-2.32) 0.96$ & $0.74(0.28-1.92) 0.74$ \\
\hline Metabolic Syndrome & $1.46(0.89-2.37) 0.12$ & $1.45(0.75-2.79) 0.26$ & $1.05(0.57-1.94) 0.85$ & $1.12(0.45-2.78) 0.79$ \\
\hline Obesity & $1.52(0.92-2.51) 0.09$ & $1.44(0.86-2.41) 0.15$ & $2.89(1.55-5.30) 0.001$ & $2.85(1.51-5.40) 0.001$ \\
\hline SAF $p>75$ & $1.49(0.91-2.43) 0.11$ & $1.81(0.88-3.71) 0.10$ & $1.50(0.77-2.92) 0.22$ & $0.46(0.16-1.30) 0.14$ \\
\hline VAF $p>75$ & $1.29(0.77-2.15) 0.37$ & $1.15(0.60-2.22) 0.66$ & $1.62(0.83-3.16) 0.15$ & $1.14(0.48-2.67) 0.75$ \\
\hline TAF $p>75$ & $1.19(0.72-1.96) 0.49$ & $0.93(0.40-2.17) 0.88$ & $1.30(0.67-2.51) 0.43$ & $0.38(0.13-1.14) 0.08$ \\
\hline Increased IMT & $2.38(1.35-4.10) 0.003$ & $2.62(1.47-4.60) 0.001$ & $0.81(0.32-2.04) 0.66$ & $0.78(0.30-1.99) 0.60$ \\
\hline
\end{tabular}

All associations were tested using logistic regression

Adjustment model $=$ M $1=$ age, current smoking, alcohol intake, physical activity, marital status

Adjustment model $=$ M $2=$ Model $1+$ Systolic BP, HDL-C, LDL-C, TG and Glucose

Hipertriglyceridemia $=\mathrm{TG}>150 \mathrm{mg} / \mathrm{dl}$, Hipoalphalipoproteinemia $=\mathrm{HDL}<40 \mathrm{mg} / \mathrm{dl}$ in men and $<50 \mathrm{mg} / \mathrm{dl}$ in women, SAF = Subcutaneous abdominal fat, $\mathrm{VAF}=$ Visceral abdominal fat, $\mathrm{TAF}=$ total abdominal fat and IMT $=$ Intima media thickness

indicators (such as blood pressure and cardiovascular symptoms) using the Karasek's model in a Mexican cohort ( $n=109$ nurses), found a significant association between job insecurity and cardiovascular indicators; pointing out the importance of these variables and their effects on stress in the Mexican work setting [28].

In the Malmo Preventive Project, a prospective study of 13,609 (2741 women) participants, self-reported chronic stress significantly increased the risk of suffering cardiovascular disease like ischemic heart disease or stroke events in middle-aged men. For women, the trends were similar but not significant after adjustment for unmodifiable, social, lifestyle risk factors, and biological parameters [29]. In contrast, we found a significant greater risk of increased intima media thickness present in women who reported chronic stress, and for obesity for men. However, the samples' characteristics and size were too small to investigate causality.

The underlying mechanisms by which different forms of mental stress increase the risk of obesity, particularly the abdominal phenotype, are complex. This has been ascribed to individual maladaptation to chronic stress exposure mediated by a dysregulation of related neuroendocrine axes. Alterations in the control and action of the hypothalamic-pituitary-adrenal axis may play a major role in this context, with the participation of the sympathetic and parasympathetic nervous system, systemic inflammation, or an interaction between genetic predisposition and stress. Specifically, the inability to cope with psychological stress, particularly early stress in life, ultimately leads to an increase of circulating catecholamines and cortisol, which in turn leads to loss of appetite control and increased adiposity [29], and resulting in an elevated heart rate and blood pressure, coronary constriction, accumulation of lipid in the intima, and electrical heart instability [30].

True comparisons between these studies and the one presented in this article are rather difficult for various reasons, mainly because of differences in methodology, ethnicity, cohort, and the participants' age. Our principal strength is the robust association found between chronic stress, obesity, and carotid atherosclerosis, following adjustment for established risk factors. This suggests that stress may play an independent role in the development of cardiovascular disease in the Mexican population.

The shortcomings of the study are identified as follows. First, the cross-sectional design of the GEA study does not allow us to determine causality as it relates to the effect of permanent self-perceived psychological stress on obesity. Second, the population-based study is not a representative and randomized cohort of Mexican population; however, the prevalence of overweight and obesity are similar to that reported by ENSANUT 2012 [31]. Third, we had missing data of the stress item in the first 254 subjects, and we were only able to perform the carotid artery ultrasound in 914 subjects.

\section{Conclusions}

In summary, we found that self-perceived chronic stress is an independent risk factor for obesity in men and carotid atherosclerosis in women, in the Mexican population. Our results suggest the need for further prospective studies in larger Hispanic populations addressing the potential role for psychological stress as a risk factor for cardiovascular 
disease. Confirmatory analyses of the association found especially in longitudinal settings, are also needed. Furthermore, in order to give insight as to which subcomponents of perceived stress are of importance, more elaborate measures of psychological stress are suggested.

\section{Competing interests}

The authors declare that they have no competing interests.

\section{Authors' contributions}

JOM initiated the study and held the overall responsibility. She designed the study, gathered background material, analyzed data, prepared and completed the manuscript. RPS revised the design and the data analyses as well as the article's intellectual content. CPS drafted the manuscript, revised it critically for important intellectual content and approved the final version of the manuscript. WOA contributed to the design, compiled parts of the background articles and revised the manuscript. AMU contributed to the concept, analyses, and interpretation of data. GCS contributed to acquisition of data and performed data base searches. EJG participated in the design of the study and statistical analyses. All authors read and approved the final manuscript.

\section{Acknowledgements}

This study was supported by Consejo Nacional de Ciencia y Tecnología (CONACYT) project number SALUD-2010-2-150537. The authors thank Eric Kimura Hayama MD, chief of the Department of Cardiac Tomography at the $\mathrm{INCICH}$, for his contribution with the magnetic resonance images.

Received: 28 January 2014 Accepted: 29 July 2015

Published online: 14 August 2015

\section{References}

1. Kuk Jl, Ardern Cl. Age and sex differences in the clustering of metabolic syndrome factors association with mortality risk. Diabetes Care. 2010;33:2457-61.

2. Barrington WE, Ceballos RM, Bishop SK, McGregor BA, Beresford SA: Perceived Stress, Behavior, and body mass index among adults participating in a worksite obesity prevention program, Seattle, 2005-2007. Prev Chronic Dis 2012, 9:120001.doi: http://dx.doi.org/10.5888/pcd9.120001.

3. Cohen S, Janicki-Deverts D, Miller GE. Psychological stress and disease. JAMA. 2007;289(14):1685-7.

4. Wardle J, Chida Y, Gibson L, Whitaker KI, Steptoe A. Stress and Adiposity: A meta-analysis of longitudinal studies. Obesity. 2011;19:771-8.

5. Compare A, Zarbo C, Shonin E, et al.: Emotional regulation and depression: A potential mediator between heart and mind. Cardiovascular Psychiatry and Neurology 2014, Article ID 324374.

6. Rosmond R, Dallman MF, Bjorntorp P. Stress-related cortisol secretion in men: Relationships with abdominal obesity and endocrine, metabolic and hemodynamic abnormalities. J Clin Endocrinol Metab. 1998;83:1853-9.

7. Gami AS, Witt BJ, Howard DE, Erwin PJ, Gami LA, Somers VK, et al. Metabolic syndrome and risk of incident cardiovascular events and death: a systematic review and meta-analysis of longitudinal studies. J Am Coll Cardiol. 2007:49(4):403-14.

8. Black PH. The inflammatory response is an integral part of the stress response: implications for atherosclerosis, insulin resistance, type II diabetes and metabolic syndrome. Brain Behav Inmun. 2003:17:350-64.

9. Jood K, Redfors P, Rosengren A, Blomstrand C, Jern C. Self-perceived psychological stress and ischemic stroke: a case control study. BMC Medicine. 2009;7:53. doi:10.1186/1741-7015-7-53.

10. Rosengren A, Hawken S, Ounpuu S, Sliwa K, Zubaid M, Almahmeed WA, et al. INTERHEART investigators: Association of psychosocial risk factors with risk of acute myocardial infarction in 11119 cases and 13648 controls from 52 countries (the INTERHEART study): case-control study. Lancet. 2004;364:953-62

11. Rosengren A, Tibblin G, Wilhelmsen L. Self-perceived psychological stress and incidence of coronary artery disease in middle-aged men. Am J Cardiol. 1991;68:1171-5

12. Villareal-Molina T, Posadas-Romero C, Romero-Hidalgo S, Antúnez-Arguelles E, Bautista-Grande A, et al.: The ABCA1 Gene R230C Variant is associated with decreased risk of premature coronary artery disease: The Genetics of
Atherosclerosis Disease GEA Study. PLoS ONE 2012, 7:11:e49285. doi: 10.1371/journal.pone.0049285.

13. Hernández-Avila M, Romieu I, Parra S, Hernández-Avila J, Madrigal H, Willett W, et al. Validity and reproducibility of a food frequency questionnaire to assess dietary intake of women living in Mexico City. Salud Publica Mex. 1998:40:133-40.

14. Baecke JA, Burema J, Frijters JE. A short questionnaire for the measurement of habitual physical activity in epidemiological studies. Am J Clin Nutr. 1982;36:936-42.

15. Grundy SM, Cleeman Jl, Daniels SR, Donato KA, Eckel RH, Franklin BA, et al. Diagnosis and management of the metabolic syndrome: a American Heart Association/National Heart Lung, and Blood Institute Scientific Statement [published corrections appear in Circulation 2005; 112(17): e297 and 2005; 112(17): e298]. Circulation. 2005;112(17):2735-52.

16. Sanchez-Castillo CP, Velazquez-Monroy O, Bever A, Lara-Esqueda A, Tapia-Conyer R, James WP. Anthropometry Cutoff points for predicting chronic disease in the Mexican National Health Survey. Obes Res. 2003;11:442-51.

17. The expert committee on the diagnosis and classification of diabetes mellitus: Report of the expert committee on the diagnosis and classification of diabetes mellitus. Diabetes Care 2003, 26 (Suppl 1): S5-S20 [No authors listed].

18. DeLong DM, DeLong ER, Wood PD, Lippel K, Rifkind BM. A comparison of methods for the estimation of plasma low- and very low-density lipoprotein cholesterol. The Lipid Research Clinics Prevalence Study. JAMA. 1986;256:2372-7.

19. Matthews DR, Hosker JP, Rudenski AS, Naylor BA, Treacher DF, Turner RC. Homeostasis model assessment: insulin resistance and beta-cell function from fasting plasma glucose and insulin concentrations in man. Diabetologia. 1985;28:412-9.

20. Mautner GC, Mautner SL, Froehlich J, Feuerstein IM, Proschan MA, Roberts WC, et al. Coronary artery calcification: assessment with electron beam CT and histomorphometric correlation. Radiology. 1994;192:619-23.

21. Kvist H, Chowdhury B, Grangard U, Tylen U, Sjostrom L. Total and visceral adipose-tissue volumes derived from measurements with computed tomography in adult men and women: predictive equations. Am J Clin Nutr. 1988;48:1351-61.

22. Longo RRC, Ricci C, Masutti F, Vidimari R, Croce LS, Bercich L, et al. Fatty infiltration of the liver. Quantification by $1 \mathrm{H}$ localized magnetic resonance spectroscopy and comparison with computed tomography. Invest Radiol. 1993;28:297-302

23. Stein JH, Korcarz CE, Hurst RT, Lonn E, Kendall CB, Mohler ER, et al. Use of carotid ultrasound to identify subclinical vascular disease and evaluate cardiovascular disease risk: A consensus statement from the American Society of Echocardiography Carotid Intima Media Thickness Task Force Endorsed by the Society for Vascular Medicine Journal of the American Society of Echocardiography. 2008. doi:10.1016/j.echo.2007.11.011.

24. Kaur J. A comprehensive review on Metabolic Syndrome. Cardiology Research and Practice 2014, http://dx.doi.org/10.1155/2014/943162.

25. Chrousos GP. Stress and disorders of the stress system. Nat Rev Endocrinol. 2009;5:374-81

26. Novak M, Bjorck L, Giang KW, Heden-Stahl C, Wilhelmsen L, Rosengren A Perceived stress and incidence of Type 2 diabetes: a 35-years follow up study of middle-aged Swedish men. Diabet Med. 2013;30:e8-e16.

27. Wolff B, Grabe HJ, Volzke H, Ludemann J, Kessler C, Dahm JB, et al. Relation between psychological strain and carotid atherosclerosis in a general population. Heart. 2005;91:460-4.

28. Juárez-García A. Psychosocial work factors associated to blood pressure and cardiovascular symptoms among Mexican nurses. Salud Publica Mex. 2007:49:109-17.

29. Ohlin B, Nilsson PM, Nilsson JA, Berglund G. Chronic psychosocial stress predicts long-term cardiovascular morbidity and mortality in middle-agged men. Eur Heart J. 2004;25:867-73.

30. Holvoet P. Stress in obesity and associated metabolic and cardiovascular disorders. Scientifica 2012, Article ID 205027.

31. Gutiérrez JP, Rivera-Dommarco J, Shamah-Levy T, Villalpando-Hernández S, Franco A, Cuevas-Nasu L, et al. Encuesta Nacional de Salud y Nutrición 2012. Mexico: Resultados Nacionales. Instituto Nacional de Salud Publica; 2012. 